gen dem Grundsatz des gegenseitigen Vertrauens folgend ohne Weiteres anerkannt werden. Sie warb für eine Vereinheitlichung von Standards für Kindesanhörungen - mindestens europaweit, idealiter international. Sie bemängelte zudem, dass das HKÜ nur Rückführungsfragen regeln würde und Regelungen zu den in diesen Verfahren geschlossenen Vereinbarungen fehlen würden; gerade diese müssten belastbar sein.

In der Schlussdiskussion mit allen Referentinnen des Tages hatten wir vor, über weiteren gesetzlichen Handlungsbedarf zu diskutieren. Tatsächlich konzentrierten wir uns dann auf das Für und Wider zum Wechselmodell. Wer einen „Glaubenskrieg“ erwartet hatte, wurde allerdings enttäuscht. Die emotionale Diskussion zeichnete sich dadurch aus, dass die Befürworterinnen und Gegnerinnen sich verstehen wollten und sich deshalb zuhörten. Hier ist insbesondere der französischen Kollegin Dank zu sagen, die mit dem Einblick in die französische Praxis zu relativieren vermochte. Als ein Beispiel mag ihr Bericht einer Anordnung des sog. „Nestmodells“ dienen. Ein Paar mit acht Kindern trennte sich und nur ein Elternteil - konkret die Mutter - war in der Lage, eine entsprechend große Wohnung zu unterhalten. Gegen den Willen der Eltern entschied sie, dass das Nestmodell versucht werden sollte. Es scheint geklappt zu haben, denn sie wurde nicht erneut befasst.

\section{Fazit}

Das deutsche Kindschaftsrecht ist im europäischen Kontext nicht von „vorgestern“. Mit Blick auf alternative - paritätische - Betreuungsmodelle und deren richterlicher Anordnung bedarf es jedoch ergänzender Überlegungen, da de lege lata die Zulässigkeit einer solchen Anordnung - wie mit nachvollziehbaren Gründen vertreten wird - zweifelhaft ist. Andererseits darf nicht verkannt werden, dass die Instanzrechtsprechung das geltende Recht für ausreichend erachtet, wenn auch mit unterschiedlicher Begründung. Ob gesetzgeberischer Handlungsbedarf besteht, sollte daher nicht vorschnell beantwortet werden, zumal (notwendige) Folgeänderungen, wie z.B. die Vertretungsbefugnis eines Elternteils bei der Geltendmachung von Unterhaltsansprüchen, mit in den Blick zu nehmen sind.

Am Ende des lebhaften Seminartages bestand Einigkeit, dass die tatsächliche Umsetzung der gemeinsamen Sorge lebbar sein muss, orientiert am allgemeinen Kindeswohl. Konkret kann dies auch durch ein Wechselmodell bzw. eine paritätische Doppelresidenz realisiert werden. Hierzu sollten die gesetzlichen Grundlagen geschaffen werden. Die Diskussion in Bonn hat uns Hoffnung gemacht, dass dies gelingen kann. Wie so oft in familienrechtlichen Streitfällen gibt es nicht die Lösung sondern immer nur eine für den konkreten Einzelfall.

\title{
Der Europäische Gerichtshof für Menschenrechte und die Rechte von Frauen'
}

Urteile können Rechtsgeschichte schreiben, auch Frauenrechtsgeschichte. Frauen haben über Jahrzehnte hin ihre Konflikte, ihre Sorgen und Nöte dem Europäischen Gerichtshof für Menschenrechte (EGMR) unterbreitet. In wichtigen Fragen haben sie Gehör gefunden, manchmal aber sind sie auch unterlegen. Der historische Bogen spannt sich von dem Urteil Marckx v. Belgien aus dem Jahr 1978, ${ }^{2}$ das dazu beigetragen hat, das Nichtehelichenrecht in Europa zu revolutionieren, über das Urteil Opuz v. Türkei aus dem Jahr $2009,{ }^{3}$ das staatliche Untätigkeit gegen häusliche Gewalt gebrandmarkt hat, bis zu S.A.S. v. Frankreich aus dem Jahr 2014, ${ }^{4}$ in dem der Gerichtshof das Verbot der Vollverschleierung in der Öffentlichkeit für konventionskonform gehalten hat. In dem Urteil Rantsev v. Russland und Zypern ${ }^{5}$ werden Missbrauch und Zwangsprostitution offengelegt, in dem Urteil Siliadin v. Frankreich ${ }^{6}$ staatliche Schutzmaßnahmen gegen die Ausnutzung von illegalen Migrantinnen und damit gegen eine Form moderner Sklaverei für unzureichend befunden. Gegenwärtig anhängig vor Gericht ist die Frage, ob das Recht auf Privatleben auch das Recht umfasst, Kinder zuhause zu gebären, ob es ein Recht einer werdenden Mutter auf eine Hausgeburt gibt. ${ }^{7}$
Derartige Fälle werfen die Frage auf, ob die Rechte von Frauen eine Sonderstellung in der Rechtsprechung des EGMR einnehmen. Sind sie ein Aliud im Vergleich zu allgemeinen Menschenrechten?

Auf internationaler Ebene ist dies anerkannt; das Übereinkommen zur Beseitigung jeder Form von Diskriminierung der $\mathrm{Frau}^{8}$ baut auf der Vorstellung auf, dass die Rechte von Frauen sich von den Rechten von Männern unterscheiden und Frauen in besonderer Weise schutzbedürftig sind. Auf europäischer Ebene gibt es zu diesem Ansatz keine echte Parallele.

1 Der Aufsatz gibt ausschließlich die persönliche Meinung der Verfasserin wieder.

2 EGMR Urt. v. 13.6.1979, Marckx v. Belgien, BeschwerdeNr. 6833/74, NJW 1979, 2449.

3 EGMR Urt. v. 9.6.2009, Opuz v. Türkei, BeschwerdeNr. 33401/02.

4 EGMR Urt. v. 1.7.2014, S.A.S. v. Frankreich, BeschwerdeNr. 43835/11.

5 EGMR Urt. v. 7.1.2010, Rantsev v. Russland und Zypern, Beschwerde-Nr. 25965/04, NJW 2010, 3003.

6 EGMR Urt. v. 26.7.2005, Siliadin v. Frankreich, Beschwerde-Nr. 73316/01, NJW 2007, 41.

7 EGMR Dubská v. Tschechien, Beschwerde-Nr. 28859/11.

8 Convention on the Elimination of All Forms of Discrimination Against Women (CEDAW), in Kraft getreten am 3.9.1981, von Deutschland am 10.7.1054 ratifiziert.
Prof. Dr. Dr. h.c. Angelika Nußberger

M.A.

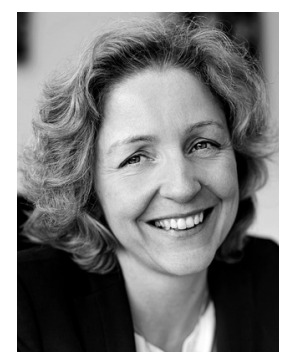

Richterin am Europäischen Gerichtshof für Menschenrechte, Straßburg/Köln 
Die Europäische Konvention zum Schutz der Menschenrechte und Grundfreiheiten (EMRK) schützt die Rechte aller, gleich ob Frauen oder Männer, grundsätzlich gleichermaßen. Explizit Erwähnung findet das Geschlecht nur in Art. 14 im Rahmen des Diskriminierungsverbots sowie in Art. 12, wenn es heißt, dass Männer und Frauen im heiratsfähigen Alter das Recht haben, eine Ehe zu einzugehen. ${ }^{9}$ Blickt man allerdings unter die sprachliche Oberfläche und analysiert die Rechtsprechung des Gerichtshofs, so zeigt sich doch, dass nicht nur die Rechte von Frauen, sondern auch die gesellschaftlichen Koordinaten, die die Rolle der Frau in der Gesellschaft bestimmen, ein Thema sind, mit dem der Gerichtshof in besonderer Weise, manchmal mit äußerster Vorsicht und Zurückhaltung, ${ }^{10}$ manchmal mit einem gewissen „Draufgängertum “ in der Annahme, alles richtig zu machen, ${ }^{11}$ umgeht.

Dies gilt es im Folgenden aufzudecken, wobei sich einerseits das Postulat, Männer und Frauen seien völlig gleich zu behandeln, als roter Faden durch die Rechtsprechung zieht, dem aber andererseits auch kontextabhängige Judikate mit einer Forderung nach einem besonderen Schutz von Frauen gegenüberstehen. Besonders spannend ist die Entwicklung der Rechtsprechung zu Kinder betreffenden Sorge- und Umgangsrechten.

\section{I) Die Auseinandersetzung des EGMR mit tradierten Rollenverständnissen}

Die Auseinandersetzung des EGMR mit tradierten Rollenverständnissen weist eine interessante Entwicklung auf, die sich an der Rechtsprechung zu unmittelbaren, unter Art. 14 EMRK subsumierbaren Ungleichbehandlungen aufzeigen lässt. Klassische Diskriminierungsfälle, in denen Frauen sich gegen Benachteiligung und Unrecht wenden, gibt es nur wenige. Ein berühmtes Beispiel ist der Fall Schuler-Zgraggen v. Schweiz ${ }^{12}$ aus dem Jahr 1993. Hier hat der Gerichtshof den als „Lebenserfahrung“ eingeführten und von den Gerichten ohne Prüfung akzeptierten Sachvortrag, Frauen unterbrächen nach der Geburt ihre Berufstätigkeit und nähmen sie erst später wieder auf, als Verstoß gegen das Gleichbehandlungsgebot im Prozessrecht, Art. 6 in Verbindung mit Art. 14 EMRK, beurteilt. Die Beschwerdeführerin war eindeutig benachteiligt worden, da sie aufgrund dieser Regelung eine Invaliditätsrente bekam, die geringer war als diejenige, die einem Mann in einer vergleichbaren Situation zugebilligt worden wäre. Bereits einige Jahre zuvor, im Jahr 1985, hatte der Gerichtshof eine ähnliche Regelung für konventionswidrig erklärt, die auf der Prämisse von einer unterschiedlichen Beteiligung von Männern und Frauen am Arbeitsmarkt aufbaute und es dementsprechend aus immigrationspolitischen Gründen Männern - im Gegensatz zu Frauen - nicht ermöglichte, ihren Ehepartnerinnen ins Ausland an die Stätte des Arbeitsplatzes nachzufolgen. ${ }^{13}$ Geklagt hatten in diesem Fall aber nicht die Frauen, sondern die Ehemänner, denen die Einreise versagt wurde.

Auch spätere Leitentscheidungen zum Diskriminierungsverbot betrafen in der Regel ${ }^{14}$ Männer, die Frauen gewährte Privilegien angegriffen, etwa die Freistellung von Frauen von der Zahlung einer Feuerwehrabgabe ${ }^{15}$, die Männern verweigerte Witwerrente $^{16}$, die in der Praxis fast ausschließlich Männern auferlegte Pflicht, das Schöffenamt zu übernehmen ${ }^{17}$ oder das nur Frauen gewährte Recht auf Elternurlaub. ${ }^{18}$ In dem Urteil zur Feuerwehrabgabe Karlheinz Schmidt gegen Deutschland aus dem Jahr 1994 vermeidet es der Gerichtshof, sich mit der Prämisse der Privilegierung, Frauen seien für den Kampf gegen das Feuer, der körperliche Kraft fordere, weniger geeignet als Männer, auseinanderzusetzen. Die deutsche Regierung hatte vorgetragen, die physischen und mentalen Besonderheiten rechtfertigten eine entsprechende Frauen begünstigende Regelung. ${ }^{19} \mathrm{Ob}$ diese Argumentation tragfähig sein könne, lässt der Gerichtshof offen ${ }^{20}$ und stellt darauf ab, dass jedenfalls Kompensationszahlungen für einen nicht geleisteten Dienst Frauen und Männer in gleicher Weise beträfen. ${ }^{21}$

9 Zusätzlich ist im - von Deutschland nicht ratifizierten - Siebten Zusatzprotokoll ein explizites Gleichbehandlungsgebot von Ehepartnern bei Ehe und Scheidung normiert, dem allerdings in der Rechtsprechung des Gerichtshofs keine praktische Bedeutung zukommt; vgl. dazu Christoph Pettiti, L'égalité entre époux, in: Frédéric Krenc, Michel Puéchavy (Hg.), Le droit de la famille à l'épreuve de la Convention des droits de l'homme, Bruxelles 2008, S. 29 ff.

10 So vermeidet der Gerichtshof oftmals, wenn er bereits eine Konventionsverletzung festgestellt hat, sich zusätzlich noch mit der Diskriminierungsfrage nach Art. 14 EMRK auseinanderzusetzen.

11 Vgl. z.B. die Kammerentscheidung im Fall Markin v. Russland (EGMR Urt. v. 7.10.2010, Beschwerde-Nr. 30078/06), die in Russland eine dezidiert negative Reaktion auslöste und zu einer Verweisung an die Große Kammer führte.

12 EGMR Urt. v. 24.6.1993, Schuler-Zgraggen v. Schweiz, BeschwerdeNr. $14518 / 89$.

13 EGMR Urt. v. 28.5.1985, Abdulaziz u.a. v. Vereinigtes Königreich, Beschwerde-Nr. 9214/80; 9473/81; 9474/81.

14 Im Namensrecht waren Beschwerdeführer in der ersten Leitentscheidung Ehepartner; vgl. EGMR Urt. v. 22.2.1994 Burghartz v. Schweiz, Series A Nr. 280-B; im Fall EGMR Urt. v. 16.2.2004, Ünal Tekeli v. Türkei, Beschwerde-Nr. 29865/96 war dagegen eine Frau Beschwerdeführerin.

15 EGMR Urt. v. 18.7.1994, Karlheinz Schmidt v. Deutschland, Beschwerde-Nr. 13580/88.

16 EGMR Urt. v. 11.6.2002, Willis v. Vereinigtes Königreich, BeschwerdeNr. 36042/97.

17 EGMR Urt. v. 20.6.2006, Zarb Adami v. Malta, BeschwerdeNr. 17209/02; interessant ist hier, dass der Gerichtshof trotz einer geschlechtsneutralen gesetzlichen Regelung eine dem entgegengesetzte, statistisch nachweisbare Praxis für eine Diskriminierung ausreichen lässt.

18 EGMR Urt. v. 27.3.1998, Petrovic v. Österreich, BeschwerdeNr. 20458/92; EGMR Urt. v. 22.3.2012, Markin v. Russland, Beschwerde-Nr. 30078/06.

19 Vgl. dazu die Argumentation der Bundesregierung (EGMR Urt. v. 18.7.1994, Karlheinz Schmidt v. Deutschland, BeschwerdeNr. 13580/88, Rn. 27): “The Government maintained that, in making this duty compulsory solely for the male sex, the legislature had taken account of the specific requirements of service in the fire brigade and the physical and mental characteristics of women. The sole aim which it had pursued in this respect was the protection of women."

20 Anders die abweichenden Meinungen der Richter Spielmann und Gotchev, die die unterschiedliche physische Kraft von Männern und Frauen für einen vernünftigen Unterscheidungsgrund halten.

21 EGMR Urt. v. 18.7.1994, Karlheinz Schmidt v. Deutschland, Beschwerde-Nr. 13580/88, Rn. 28: "Irrespective of whether or not there can nowadays exist any justification for treating men and women differently as regards compulsory service in the fire brigade, what is finally decisive in the present case is that the obligation to perform such service is exclusively one of law and theory. In view of the continuing existence of a sufficient number of volunteers, no male person is in practice obliged to serve in a fire brigade. The financial contribution has - not in law but in fact - lost its compensatory character and has become the only effective duty. In the imposition of a financial burden such as this, a difference of treatment on the ground of sex can hardly be justified." 
Wenige Jahre später, im Jahr 1998, gilt es über die Konventionskonformität des in Österreich Frauen, aber auch nur Frauen gewährten Privilegs, nach der Geburt des Kindes und nach dem Ende der Mutterschutzzeit Elternurlaub zu nehmen, zu befinden. ${ }^{22}$ Auch dieses Privileg baut erkennbar auf einem bestimmten Rollenverständnis auf, nach dem die Mutter das Kind nicht nur zur Welt bringt, sondern auch für die Versorgung im Kleinkindesalter primär zuständig ist. ${ }^{23}$ Der Gerichtshof widerspricht dem mit einer sehr vorsichtigen Formulierung:

"Der Gerichtshof ist sich dessen bewusst, dass Unterschiede zwischen Mutter und Vater in ihrer Beziehung zum Kind bestehen können, geht aber von der Annahme aus, dass, soweit es darum geht, sich um das Kind im relevanten Zeitraum zu kümmern, beide Eltern 'gleich gestellt' sind." 24

Das von dieser Annahme abweichende Rollenverständnis, das der österreichischen Gesetzgebung zugrunde liegt, wird aber, anders als von der Kommission vorgeschlagen, nicht für unvereinbar mit der Konvention erklärt. Vielmehr entscheidet der Gerichtshof den Fall Petrovic v. Österreich aufgrund von Überlegungen zur Berechtigung von schrittweise durchgeführten und im europäischen Kontext innovativen Reformen. Auf dieser Grundlage kommt er zu dem Ergebnis, Österreich sei berechtigt, eine entsprechend „frauenfreundliche“ Maßnahme einzuführen, ohne gegen die Konvention zu verstoßen, da andere Konventionsstaaten in diesem Bereich noch überhaupt keine gesetzgeberischen Maßnahmen getroffen hätten. Dem liegt zugrunde, dass bei derartigen Reformen ein diskriminierungsfreies Alles-oder-Nichts-Prinzip als kontraproduktiv angesehen wird.

Anders ist der Ton des Gerichtshofs dagegen im Jahr 2012, als er nochmals dazu aufgerufen ist, sich mit einer derartigen Fragestellung zu befassen. Diesmal geht es um die in Russland nur weiblichen, nicht aber männlichen Militärangehörigen gewährte Vergünstigung, Elternurlaub zu nehmen. Nunmehr greift der Gerichtshof das von der russischen Regierung zur Verteidigung angeführte traditionalistische Familienverständnis unmittelbar an. Die russische Regierung hatte sich auf wissenschaftliche Erkenntnisse berufen, die zeigten, dass zwischen Mutter und Neugeborenem eine besondere psychologische Beziehung existiere; daher sei die Anwesenheit der Mutter zuhause im ersten Lebensjahr des Kindes von besonderer Bedeutung. ${ }^{25}$ Dem widersprach die Große Kammer des Gerichtshofs mit einer leichten Variation seiner aus dem Jahr 1998 stammenden Aussage im Fall Petrovic. Hatte er damals noch zurückhaltend formuliert, dass er „davon ausgehe“ (,starts from the premise“), dass Männer und Frauen bei der frühkindlichen Erziehung gleichgestellt seien, präsentiert er dies nunmehr als Schlussfolgerung („concludes“). Das Urteil der Kammer hatte dies noch wesentlich deutlicher auf den Punkt gebracht:

„Soweit die Unterscheidung auf traditionelle Geschlechterrollen gestützt wird, d.h. auf die Annahme, Frauen seien primär für die Sorge für die Kinder verantwortlich und Männer primär für den Broterwerb, können diese an das Geschlecht anknüpfenden Vorurteile vom Gericht nicht als ausreichende Rechtfertigung für eine unterschiedliche Behandlung angesehen werden, ebenso wenig wie ähnliche Vorurteile, die auf Rasse, Herkunft, Hautfarbe und sexuelle Orientierung gründen. “26
Mit der Entscheidung im Fall Petrovic wurde den Mitgliedsstaaten auch unter Berufung auf den nationalen Ermessensspielraum eine Übergangszeit zur Beseitigung von mit Rollenklischees verbundenen, die Frauen vorgeblich schützenden Regelungen eingeräumt und Gleichberechtigung insoweit als Prozess angesehen. ${ }^{27}$ Dieser Prozess darf, wie das Urteil Markin v. Russische Föderation aus dem Jahr 2012 zeigt, nunmehr als abgeschlossen gelten.

Anders ist dies aber im Rentenrecht, etwa bei der unterschiedlichen Festsetzung von Rentenaltersgrenzen für Männer und Frauen, insbesondere, wenn dies zum Ausgleich einer historisch nachweisbaren - besonderen Belastung von Frauen durch Kindererziehung und Beruf dienen soll. Im Fall Andrle v. Tschechische Republik aus dem Jahr 2011 setzt sich der Gerichtshof explizit mit den entsprechenden Rollen der Geschlechter in der Tschechoslowakei in den 1960er Jahren auseinander und rechtfertigt Privilegien von Frauen bei der Bestimmung des Rentenalters als „affirmative action“. Wie lange diese rechtfertigbar sei, sei nicht eindeutig zu bestimmen:

„Dennoch ist es schwierig, genau den bestimmten Augenblick auszumachen, an dem die unfaire Behandlung der Männer die Notwendigkeit, die benachteiligte Position der Frauen durch affirmative action zu korrigieren, aufwiegt. " 28

Unterbrechen Frauen wegen der Kindererziehung ihre berufliche Tätigkeit, so rechtfertigt auch dies Begünstigungen im Rentenrecht. Väter mit durchgängigen Erwerbsbiographien, die geltend machten, sie hätten keine Chance auf einer Kinder-Auszeit gehabt, aber auch nicht nachweisen konnten, es versucht zu haben, konnten in Straßburg mit ihren Beschwerden nicht durchdringen:

„Im Übrigen können die Beschwerdeführer ihre Situation nicht mit derjenigen ihrer Amtskolleginnen vergleichen, da eben jene Unterbrechung der Tätigkeit mit den negativen Auswirkungen auf die Entwicklung ihrer Karriere und die Berechnung ihrer Renten fehlt. “29

22 EGMR Urt. v. 27.3.1998, Petrovic v. Österreich, BeschwerdeNr. 20458/92.

23 Dies wird von der Regierung explizit als allgemeines gesellschaftliches Verständnis hervorgehoben: „Furthermore, the provisions in question reflected the outlook of society at the time, according to which the mothers had the primary role in looking after young children." (EGMR Urt. v. 27.3.1998, Petrovic v. Österreich, Beschwerde-Nr. 20458/92, Rn. 32).

24 EGMR Urt. v. 27.3.1998, Petrovic v. Österreich, BeschwerdeNr. 20458/92, Rn. 36.

25 EGMR Urt. v. 22.3.2012, Markin v. Russland, BeschwerdeNr. 30078/06, Rn. 116.

26 EGMR Urt. v. 07.10.2010, Markin v. Russland, Kammerentscheidung, Beschwerde-Nr. 30078/06, Rn. 58.

27 "Originally, welfare measures of this sort - such as parental leave were primarily intended to protect mothers and to enable them to look after very young children. Only gradually, as society has moved towards a more equal sharing between men and women of responsibilities for the bringing up of their children, have the Contracting States introduced measures extending to fathers, like entitlement to parental leave." (EGMR Urt. v. 27.3.1998, Petrovic v. Österreich, Beschwerde-Nr. 20458/92, Rn. 40).

28 EGMR Urt. v. 17.2.2011, Andrle v. Tschechische Republik, BeschwerdeNr. 6268/08, Rn. 56.

29 EGMR Urt. v. 15.10.2013, Ryon u.a. v. Frankreich, BeschwerdeNr. 33014/08, 11793/09, 36748/08, 43329/10, 5187/09, 66405/10, Rn. 44. 
Ein interessanter Aspekt bei der Auseinandersetzung mit auf bestimmten Rollenverständnissen beruhenden Schutzkonzepten von Frauen ist auch dem Urteil S.A.S. v. Frankreich, ${ }^{30}$ dem berühmten „Burka-Urteil“, zu entnehmen. Die französische Regierung hatte argumentiert, das Verbot der Vollverschleierung diene der Würde der Frau und ihrem Schutz vor Diskriminierung, Argumente, die auch in der allgemeinen gesellschaftlichen Debatte oft vorgetragen wurden, im Grunde aber der Frau das Recht, eigenständig ihre Rolle in der Gesellschaft, und sei es eine gewillkürte Unterwerfung unter den Mann, zu bestimmen, aberkannten. Dem hat sich der Gerichtshof mit klaren Worten entgegengestellt und erklärt, man könne nicht vor der Ausübung der eigenen Grundrechte geschützt werden. ${ }^{31}$

Nichtsdestotrotz erklärte die Mehrheit des Gerichtshofs das Burka-Verbot für kompatibel mit der Konvention, da es dazu diene, das Miteinander-Leben sicherzustellen, für das es nötig sei, das Gesicht zu zeigen und zur Kommunikation bereit zu sein. Damit bekennt sich der Gerichtshof aber nicht nur zu einem bestimmten Gesellschaftsmodell, sondern verwehrt jenen, die sich - vorgeblich oder tatsächlich von der Religion bestimmt - in dieses Modell nicht einpassen wollen, den Schutz. Dabei wird der Schleier undifferenziert als Symbol der Unterwerfung gesehen und dies, obwohl die Beschwerdeführerin explizit erklärt hatte, selbst zu bestimmen, ob und wann sie die Burka tragen wolle und sich damit auf ihre Weise zu emanzipieren. Diesen Emanzipationsweg schneidet der Gerichtshof ab, da er insoweit die „Wahl der Gesellschaft “32 anerkennt. $^{33}$

Streitigkeiten um religiöse Bekleidungsregeln haben allemal einen geschlechterspezifischen Kontext, ${ }^{34}$ den der Gerichtshof aber in den meisten Entscheidungen ausklammert und sich auf den Konflikt zwischen Religionsfreiheit und Recht auf Privatleben konzentriert.

Explizit angesprochen wird der Aspekt der Diskriminierung aufgrund des Geschlechts aber im Fall Dahlab v. Schweiz; wenn auch die Ausführungen vergleichsweise kursorisch sind:

"Der Gerichtshof stellt im vorliegenden Fall fest, dass die Maßnahme, mit der der Beschwerdeführerin ausschließlich im Kontext ihrer beruflichen Pflichten verboten wurde, das islamische Kopftuch zu tragen, nicht gegen sie als Mitglied des weiblichen Geschlechts gerichtet war, sondern das legitime Ziel verfolgte, die Neutralität des Staates im Grundschulerziehungssystem sicherzustellen. Eine derartige Maßnahme könnte auch auf einen Mann angewendet werden, der unter ähnlichen Umständen Kleidung trüge, die ihn eindeutig als Mitglied einer anderen Religion identifizieren würde. “35 Geht es um die Rechtfertigung einer unterschiedlichen Behandlung von Männern und Frauen, wird oftmals auch auf die Biologie abgestellt. Altersgrenzen bei der Gebärfähigkeit hat der Gerichtshof nicht als tauglichen Unterscheidungsgrund angesehen. ${ }^{36}$ Wohl aber hat er es dem Gesetzgeber zuerkannt, auf die Unfähigkeit lesbischer Paare, Kinder zu bekommen, abzustellen. In dem Fall Böckel und Gessner-Böckel v. Deutschland ${ }^{37}$ machten zwei lesbische Frauen geltend, auch zu ihren Gunsten müsse eine $\mathbb{} 1592$ Abs. 1 BGB entsprechende „Vaterschaftsvermutung “ in Ansatz gebracht werden können, mit der Folge dass das von einer Frau zur Welt gebrachte Kind automatisch als Kind auch der mit ihr in eingetragener Lebensgemeinschaft lebenden Partnerin gilt und bereits vor einer Adoption eine entsprechende Eintragung in die Geburtsurkunde des Kindes vorgenommen wird. Dem ist der EGMR nicht gefolgt; er hält die Situation von heterosexuellen und lesbischen Paaren insoweit für nicht vergleichbar.

Allgemein lässt sich festhalten, dass der EGMR Probleme mit feinem Sensor und einem auf der ratio beruhenden Argumentationsmuster aufdeckt, indem er für eine unterschiedliche Behandlung von Männern und Frauen nicht nur „vernünftige“, sondern „,besonders schwerwiegende Gründe“ (,very weighty reasons“) fordert. ${ }^{38}$ Eine politisch-aufklärerische Rolle übernimmt er, soweit er auf die Förderung der Geschlechtergleichheit als politische Priorität des Europarats verweist und sie in seine Argumentation als „zentrales Prinzip der Konvention “39 integriert. ${ }^{40}$ Auf dieser

30 EGMR Urt. v. 1.7.2014, S.A.S. v. Frankreich, Beschwerde-Nr. 43835/11.

31 EGMR Urt. v. 1.7.2014, S.A.S. v. Frankreich, Beschwerde-Nr. 43835/11, Rn. 119: „The Court takes the view, however, that a State Party cannot invoke gender equality in order to ban a practice that is defended by women - such as the applicant - in the context of the exercise of the rights enshrined in those provisions, unless it were to be understood that individuals could be protected on that basis from the exercise of their own fundamental rights and freedoms."

32 EGMR Urt. v. 1.7.2014, S.A.S. v. Frankreich, Beschwerde-Nr. 43835/11, Rn. 153: „Furthermore, admittedly, as the applicant pointed out, by prohibiting everyone from wearing clothing designed to conceal the face in public places, the respondent State has to a certain extent restricted the reach of pluralism, since the ban prevents certain women from expressing their personality and their beliefs by wearing the full-face veil in public. However, for their part, the Government indicated that it was a question of responding to a practice that the State deemed incompatible, in French society, with the ground rules of social communication and more broadly the requirements of "living together". From that perspective, the respondent State is seeking to protect a principle of interaction between individuals, which in its view is essential for the expression not only of pluralism, but also of tolerance and broadmindedness without which there is no democratic society (see paragraph 128 above). It can thus be said that the question whether or not it should be permitted to wear the full-face veil in public places constitutes a choice of society."

33 Vgl. dazu das Sondervotum der Richterinnen Nußberger und Jäderblom zu EGMR Urt. v. 1.7.2014, S.A.S. v. Frankreich, Beschwerde-Nr. 43835/11.

34 Vgl. dazu Angelika Nußberger, Unauflösbare

Wertungswidersprüche? Die Rechtsprechung des Europäischen Gerichtshofs für Menschenrechte zur Autonomie von Religionsgemeinschaften und zum Verbot der Diskriminierung aufgrund des Geschlechts, in: Ute Mager/Juliane Kokott, Religionsfreiheit und Gleichberechtigung der Geschlechter, Tübingen 2014 S. 161-178.

35 EGMR Urt. v. 15.2.2001, Dahlab v. Schweiz, BeschwerdeNr. 42393/98, NJW 2001, 2871-2873.

36 EGMR Urt. v. 21.2.1997, Von Raalte v. Niederlande, BeschwerdeNr. 20060/92.

37 EGMR Entsch. v. 7.5.2013, Böckel und Gessner-Böckel v. Deutschland, Beschwerde-Nr. 8017/11.

38 So erstmals in dem Urteil im Fall Abdulaziz v. Vereinigtes Königreich aus dem Jahr 1985 (FN 10). In dem Urteil Schuler-Zgraggen aus dem Jahr 1993 hatte der Gerichtshof noch ,any reasonable and objective justification“ ausreichen lassen. In dem Urteil van Raalte v. die Niederlande spricht er dagegen von "compelling reasons“; vgl. zu den entsprechenden Anforderungen in rechtsvergleichender Perspektive Anne Peters, Doris König, Das Diskriminierungsverbot, in: Dörr, Grote, Marauhn (Hg.), EMRK/GG. Konkordanzkommentar, 2. Auflage, Tübingen 2013, Band 2 Rd. 135 ff.

39 EGMR Urt. v. 10.11.2005, Leyla Sahin v Türkei, BeschwerdeNr. 44774/98, Rn. 107.

40 EGMR Urt. v. 24.6.1993, Schuler-Zgraggen v. Schweiz, BeschwerdeNr. 14518/89, Rn. 67, EGMR Urt. v. 22.3.2012, Markin v. Russland, Beschwerde-Nr. 30078/06, Rn. 127. 
Linie liegt es auch, dass der Gerichtshof eine politische Partei, die eine Ungleichbehandlung von Männern und Frauen beim Wahlrecht fordert und die Männer als den „Kopf“ der Frauen bezeichnet, beim Gerichtshof mit ihrer auf die Religionsfreiheit gegründeten Beschwerde kein Gehör finden konnte; die Kammer sah eine Prüfung nicht als notwendig an und wies die Beschwerde als unzulässig $a b .{ }^{41}$ Dabei zitierte sie die vom niederländischen Obersten Gericht verwendete Formel, nach der die Position der immerhin seit 1922 im Unterhaus vertretenen Partei ,inakzeptabel sei, ganz gleich auf welchen tiefen religiösen Überzeugungen sie gegründet sein mag“. ${ }^{42}$ Ein selten klares Wort. ${ }^{43}$

\section{II) Anerkennung einer besonderen Schutzbedürftigkeit von Frauen}

Wahr ist aber auch, dass der Gerichtshof für Frauen in bestimmten Situationen besonderen Schutz und besondere Fürsorge fordert. Auch dies ist ein wichtiger Strang in der Rechtsprechung des EGMR zu den Rechten von Frauen.

Ein in diesem Zusammenhang relevanter Aspekt ist die Berücksichtigung des Geschlechts bei der Feststellung, ob eine „unmenschliche Behandlung “ vorliegt - hier kann die Schwelle bei Frauen aufgrund ihrer physischen Konstitution und ihrer besonderen Verletzbarkeit niedriger anzusetzen sein. So lautet die Standardformel folgendermaßen:

„Die Beurteilung dieses Minimums ist, wie es in der Natur der Sache liegt, relativ; es hängt von allen Umständen des Falles ab wie der Dauer einer Misshandlung, den physischen und psychischen Wirkungen und, in manchen Fällen, dem

Geschlecht, Alter und Gesundheitszustand des Opfers etc. “"44 Gerade auch bei der Abgrenzung zwischen „unmenschlicher Behandlung“ und „Folter“ kann der Aspekt, dass Frauen in der Untersuchungshaft unter Umständen mehreren Männern schutzlos gegenüberstehen, eine Rolle spielen, wie der Fall Aydin v. Türkei ${ }^{45}$, der eine Vergewaltigung betrifft, sowie der Fall Menesheva v. Russland ${ }^{46}$, bei dem es um Schläge und sonstige Misshandlungen geht, zeigen. Aus der Sicht des Gerichtshofs schlägt das Pendel dann eher in Richtung „Folter“ aus.

Eine eigene Fallgruppe unter Art. 2 und 3 EMRK bilden Ausweisungsfälle, in denen das Geschlecht mit Blick auf die den Betroffenen in ihren Heimatstaaten drohenden Gefahren für Leib und Leben eine Rolle spielen kann. So wendet der Gerichtshof etwa bei der Gefahr einer Beschneidung der weiblichen Genitalorgane Art. 3 EMRK an. ${ }^{47}$ Auch der Vortrag, allein als Frau ohne männlichen Schutz einer Lebensgefahr ausgesetzt ${ }^{48}$ oder aufgrund eines bestimmten Rollenverständnisses in der eigenen Familie oder aufgrund bestimmter moralischer Vergehen mit Gewalt konfrontiert ${ }^{49}$ zu sein, kann einer Ausweisung aus der Sicht des Gerichtshofs entgegenstehen.

In diesen Zusammenhang passt gleichfalls die Rechtsprechungslinie zu staatlichen Schutzpflichten gegenüber besonders gefährdeten Gruppen, für die insbesondere auch eine eigenständige Schutzgesetzgebung eingefordert werden kann; dass es sich dabei in aller Regel um Frauen handelt, ist offensichtlich ${ }^{50}$ und lässt auch der Gerichtshof nicht unerwähnt. ${ }^{51}$ Beispiele sind der Schutz gegen Zwangsarbeit illegaler Migrantinnen, ${ }^{52}$ der Schutz gegen sexuellen Missbrauch geistig behinderter Frauen, ${ }^{53}$ der Schutz gegen Zwangsprostitution und Menschenhandel ${ }^{54}$ sowie last but not least der Schutz gegen häusliche Gewalt. ${ }^{55}$ Auch bei Nacktaufnahmen ohne Einverständnis kann eine strafrechtliche Regelung als Abschreckung erforderlich sein. ${ }^{56}$

Auch in Abtreibungsfällen haben sich betroffene Frauen, denen eine Abtreibung verweigert wurde, auf Art 3 EMRK, das Verbot der unmenschlichen Behandlung, berufen. Diesen Ansatz hat der Gerichtshof für unzulässig erklärt. Dagegen kann es aus der Sicht des Gerichtshofs eine Verletzung des Rechts auf Privatleben (Art. 8 EMRK) darstellen, wenn einer werdenden Mutter kein Verfahren zur Überprüfung der therapeutischen Notwendigkeit einer Abtreibung zur Verfügung gestellt wird mit der Folge, dass ihre Gesundheit durch die Schwangerschaft dauerhaft geschädigt wird. ${ }^{57}$ Dies gilt auch, wenn eine Frau bei einer medizinischen Indikation gezwungen ist, den Schwanger-

41 EGMR Urt. v. 10.7.2012, Staatkundig Gereformeerde Partij v. die Niederlande, Beschwerde-Nr. 58369/10.

42 EGMR Urt. v. 10.7.2012, Staatkundig Gereformeerde Partij v. die Niederlande, Beschwerde-Nr. 58369/10.

43 Vgl. dazu ausführlich Nußberger a.a.O. (Fn. 34), S. 169 ff.

44 EGMR Urt. v. 18.1.1978, Irland v. Großbritannien, BeschwerdeNr. 5310/71, Rn. 162.

45 EGMR Urt. v. 25.9.1997, Aydin v. Türkei, Beschwerde-Nr. 23178/94.

46 EGMR Urt. v. 9.3.2006, Menesheva v. Russland, BeschwerdeNr. 59261/00.

47 EGMR Entscheidung v. 17.5.2011, Izevbekhai v. Irland, BeschwerdeNr. 43408/08; EGMR Entscheidung v. 20.9.2011, Mary Magdalene Omeredo v. Österreich, Beschwerde-Nr. 8969/10: in beiden Fällen, die Ausweisungen nach Nigeria betreffen, betont der Gerichtshof zwar, dass „Female genital mutilation“ (FGM) eine Verletzung von Art. 3 EMRK darstellen würde, erkennt aber aufgrund von Fluchtalternativen in den konkreten Fällen keine unmittelbare Gefahr für die betroffenen Frauen.

48 Vgl. z.B. EGMR Urt. v. 27.3.2014, W.H. v. Schweden (nicht rechtskräftig, Verweis an die Große Kammer), in dem es um eine geschiedene Frau aus dem Irak und eine Fluchtalternative in Kurdistan geht.

49 Vgl. z.B. EGMR Urt. v. 20.7.2010; N. v. Schweden, in dem der Gerichtshof von einer besonderen Gefährdung einer getrennt von ihrem Mann lebenden Frau aus Afghanistan aufgrund familiärer und gesellschaftlicher Racheakte ausgeht. Die Ausführungen des Gerichtshofs sind sehr allgemein: "The Court firstly observes that women are at particular risk of illtreatment in Afghanistan if perceived as not conforming to the gender roles ascribed to them by society, tradition and even the legal system.", ebd., para. 55.

50 Vgl. die in den Urteilen erwähnten ausführlichen Berichte verschiedener Institutionen wie insbesondere des Menschenrechtskommissars des Europarats, die auf die Gefährdung von Frauen aufmerksam machen. Auch die vor den Gerichtshof gebrachten Fälle betrafen alle Frauen.

51 EGMR Urt. v. 26.7.2005, Siliadin v. Frankreich, NJW 2007, 41, Rn. 88: „La Cour relève enfin qu'il ressort des constatations de l'Assemblée parlementaire (...) que " les esclaves d'aujourd'hui sont en majorité des femmes qui travaillent le plus souvent chez des particuliers, chez qui elles arrivent comme domestiques immigrées [...].“; vgl. auch Rn. 111.

52 EGMR Urt. v. 26.7.2005, Siliadin v. Frankreich, NJW 2007, 41

53 EGMR Urt. v. 26.3.1985, X und Y v. Niederlande, BeschwerdeNr. 8978/80.

54 EGMR Urt. v. 7.1.2010, Rantsev v. Russland und Zypern, BeschwerdeNr. 25965/04, NJW 2010, 3003.

55 EGMR Urt. v. 9.6.2009, Opuz v. Türkei, Beschwerde-Nr. 33401/02.

56 EGMR Urt. v. 12.11.2013, Södermann v. Schweden, BeschwerdeNr. 5786/08, NJW 2014, 607

57 EGMR Urt. v. 20.3.2007, Tysiąc v. Polen, Beschwerde-Nr. 5410/03, FamRZ 2007, 707. 
schaftsabbruch im Ausland vornehmen zu lassen. ${ }^{58}$ Allerdings hat der Gerichtshof in der kontroversen Entscheidung ABC v. Irland nicht angenommen, dass dies auch für abtreibungswillige Frauen ohne medizinische Indikation gelten würde. Auch wenn es einen europäischen Grundkonsens in diesen Fragen gäbe, so habe doch der einzelne Mitgliedsstaat einen Ermessensspielraum bei nicht juristisch entscheidbaren Fragen wie dem Beginn menschlichen Lebens. ${ }^{59}$ Die restriktive Regelung, die in Irland auf mit Referenden abgesichertem Verfassungsrecht beruhe, sei insofern zu achten. Zu Diskriminierungsfragen hat der Gerichtshof auch in diesem Zusammenhang nicht Stellung bezogen.

Die Antwort des Gerichtshofs auf die Frage, ob Frauen anders sind und daher eines besonderen Schutzes bedürfen, kann insofern mit einem „unter Umständen ja “ beantwortet werden. Sind gesetzgeberische Maßnahmen vonnöten, müssen sie nicht explizit auf Frauen abstellen, wohl aber die Situationen, in denen Frauen typischerweise gefährdet sind, mit umfassen.

\section{III) Rechtsbeziehungen zum Kind}

\section{1) Sonderrechte der Mütter aufgrund von Schwangerschaft und Geburt?}

Bekommt eine nicht verheiratete Frau ein Kind, so hat sie, sollte man meinen, (mindestens) alle Rechte, die auch verheiratete Frauen in derselben Situation haben. Heute ist dies, nimmt man einmal Leihmutterschaft aus, eine Selbstverständlichkeit. In Belgien in den 1970er Jahren dagegen musste die Mutter ihr Kind nach der Geburt zunächst anerkennen, bevor sie die Mutterrolle überhaupt übernehmen durfte. Auch dann waren ihre Rechte und die Rechte des Kindes noch eingeschränkt, etwa mit Blick auf das Erbrecht nach den Großeltern mütterlicherseits. Hier hat der Gerichtshof im Jahr 1978 mit Marckx v. Belgien ${ }^{60}$ eine Leuchtturm-Entscheidung getroffen, die nicht nur methodisch für die Auslegung der Konvention von herausragender Bedeutung ist, sondern auch die Rechte nicht verheirateter Mütter und ihrer Kinder revolutioniert hat. Dies hat noch heute Nachwirkungen, insbesondere im Erbrecht, wenn Ungleichbehandlungen, etwa beim Erbrecht nach dem Vater, aufgrund von Übergangsregelungen fortwirken. ${ }^{61}$

Die eigentlichen Probleme liegen heute aber anderswo. ${ }^{62}$ So ist nicht mehr sicher, dass die Mutter, die ein Kind zur Welt bringt, auch in jeder Hinsicht „Mutter“ ist. Die Leihmutterschaft wirft gravierende rechtliche Probleme auf, die gegenwärtig vor verschiedene internationale Gerichte gebracht werden; die Befassung damit steht noch ganz am Anfang. Der EGMR hatte sich im Fall Mennesson v. Frankreich ${ }^{63}$ mit einem bestimmten Aspekt der Problematik auseinanderzusetzen und hat erste Fragen zur Vaterschaft beantwortet. So hat er erklärt, dass es gegen das „,beste Interesse des Kindes “ verstoße, wenn auch dann, wenn die biologische Abstammung vom Vater nachgewiesen ist, keine rechtliche Möglichkeit besteht, ein rechtliches Band zum Vater zu knüpfen. Das französische Recht ist insoweit sehr streng und schließt nicht nur eine Anerkennung der Vaterschaft, sondern auch etwa eine Adoption aus. Der Mutter wurden dagegen vom Gerichtshof explizit keine Rechte zuerkannt. Sie war im konkreten Fall nicht mit den Zwillingen verwandt, da der Leihmutter die Eizelle einer dritten Frau eingesetzt worden war. Aus der Sicht des Gerichtshofs wurde zwar mit der restriktiven Regelung in das Familienleben der Eltern sowie auch der Kinder eingegriffen; dieser Eingriff war aber mit ordre-public-Überlegungen zum allgemeinen Schutz der Rechte der Kinder und der Leihmütter zu rechtfertigen. Verletzt waren dagegen die Rechte der Kinder aus Art. 8 EMRK, da das Recht auf Privatleben auch die Identität mit umfasst, für die die biologische Abstammung ein wesentliches Element ist.

\section{2) Umgangs- und Sorgerecht von Vätern}

Schwerpunkt der familienrechtlichen Rechtsprechung des Gerichtshofs im letzten Jahrzehnt war das Umgangs- und Sorgerecht der biologischen Väter im Verhältnis zu ihren Kindern, wenn die Beziehung zwischen Vater und Mutter, sei es vor, sei es nach der Geburt auseinanderbricht ohne dass sie je verheiratet waren.

Den Ausgangspunkt der Rechtsprechung und auch die Dynamik der Entwicklung illustriert gut die von der Europäischen Menschenrechtskommission noch in den 1980er Jahren abgegebene Stellungnahme, nach der davon auszugehen sei, dass Väter unehelicher Kinder nicht am Kontakt zu ihren Kindern interessiert seien und die nicht-eheliche Gemeinschaft jederzeit verlassen könnten. Dies rechtfertigte es nach Ansicht der Kommission, der Mutter Sorge- und Umgangsrecht zu übertragen. Dies gelte auch trotz der Zunahme der Zahl nichtehelicher Lebensgemeinschaften. ${ }^{64}$

Auch wenn diese Annahmen sicherlich im 21. Jahrhundert überholt sind und inzwischen bereits aus einer sehr fernen Zeit zu stammen scheinen, so zeigen sie doch nicht nur den Status quo des Rechts in den 80er Jahren, sondern auch dessen, was in der Gesellschaft für „normal“ gehalten wurde. Vor diesem Hintergrund ist es nicht überraschend, dass es einer Vielzahl von

58 EGMR Urt. v. 16.12.2010, A, B und C v. Irland, BeschwerdeNr. 25579/05, NJW 2011, 2107: Eine Verletzung wurde bei der dritten Beschwerdeführerin festgestellt, bei der aufgrund einer Krebserkrankung eine medizinische Indikation gegen die Schwangerschaft vorlag, die sich aber dennoch gezwungen sah, die Abtreibung nicht in Irland, sondern in Großbritannien vorzunehmen.

59 Mit einer Mehrheit von 11 zu 6 Stimmen wurde bei den ersten beiden Beschwerdeführerinnen im Fall A, B und C v. Irland (Fn. 58) keine Verletzung von Art. 8 und 13 EMRK festgestellt.

60 Marckx v. Belgien, Beschwerde-Nr. 6833/74, NJW 1979, 2449.

61 EGMR Urt. v. 7.2.2013, Fabris v. Frankreich, Beschwerde-Nr. 16574/08, NJW-RR 2014, 645.

62 Vgl. den auf das französische Recht bezogenen umfassenden Überblick zu den Veränderungen im Verständnis dessen, was „Familie“ ist und in diesem Zusammenhang einer Regelung bedarf : Marie Douris, La diversité des couples et l'unicité de la parenté: une évolution contenue du droit français de la famille, in: Pascale Boucaud, L'évolution du concept de famille en Europe, depuis trente ans: étude pluridisciplinarie, S. 13-58 die die Frage stellt, ob nunmehr etwas Neues entsteht, „une nouvelle assise symbolique, juridique et équilibrée de la famille, entre parenté et parentalité (eine neue symbolische, juristische und ausgeglichene Konzeption von Familie zwischen Verwandtschaft und Elternschaft), ebd. S. 15.

63 EGMR Urt. v. 26.6.2014, Mennesson v. Frankreich, BeschwerdeNr. 65192/11, FamRZ 2014, 1349; vgl. auch EGMR Urt. v. 26.6.2014, Labasse v. Frankreich, Beschwerde-Nr. 65941/11.

64 Vgl. die unveröffentlichten Kommissions-Entscheidungen zu Beschwerde Nr. 9588/81 Entscheidungen v. 15.3.1984 und zu Beschwerde Nr. 9530/81 Entscheidung v. 14.5.1984; zitiert in: EGMR Urt. v. 13.7.2000, Elsholz v. Deutschland, Beschwerde-Nr. 25735/94, NJW 2001, 2315-2319, Rn. 48. 
Klagen bedurfte, um Gesetzgebung und Rechtsanwendung auf eine neue Grundlage zu stellen. ${ }^{65}$

Die Beschwerden kamen - wie bei den Gleichberechtigungsfällen - fast ausschließlich von Männern. ${ }^{66}$ Die Liste der oftmals aus Deutschland kommenden Beschwerdeführer - Elsholz, Sommerfeld, Zaunegger, Anayo, Schneider, Kautzor und Ahrens - ist lange. Erfolgreich angegriffen wurde das Verfahren, Umgangsrechte des Vaters ohne psychologisches Expertengutachten und ohne persönliche Anhörung auszuschließen und dabei auch dann den Willen der Mutter für ausschlaggebend zu halten, wenn nachweisbar ist, dass sie zur Entfremdung zwischen Vater und Kind wesentlich beigetragen hatte. ${ }^{67}$ Gleichfalls als Konventionswidrig angesehen wurde, dass die Frauen mit ihrem Veto ein gemeinsames Sorgerecht verhindern konnten. ${ }^{68}$ Dies gilt schließlich auch für die Annahme, es sei im besten Interesse des Kindes, wenn dem biologischen Vater nur dann ein Umgangsrecht eingeräumt werde, wenn er sich bereits um das Kind gekümmert habe, nicht aber, wenn ihm der Zugang zum Kind durch die Mutter von Anfang an verweigert wurde. Aus der Sicht des Gerichtshofs muss wenigstens eine gerichtliche Überprüfung dessen, was tatsächlich im Kindeswohl ${ }^{69}$ ist, möglich sein und darf nicht eine unwiderlegbare Vermutung zur Anwendung kommen, nach der die Stabilität der rechtlichen Familie immer Vorrang haben müsse. ${ }^{70}$ Bei diesen Fällen erkannte der Gerichtshof in der Regel auf eine Verletzung der Rechte der Väter sowohl aus Art. 8 EMRK als auch - mit Blick auf das Verfahren - aus Art. 6 EMRK. Nur in wenigen Fällen wurde darüber hinaus explizit auf das Diskriminierungsverbot zu Lasten des Vaters abgestellt, etwa bei der Regelung, nach der sich die Mutter einem gemeinsamen Sorgerecht entgegenstellen konnte ohne dass dies einer gerichtlichen Überprüfung zugänglich gewesen wäre. ${ }^{71}$

\section{3) Recht auf Feststellung der Vaterschaft}

Anders hat der Gerichtshof die weitergehenden Forderungen biologischer Väter beurteilt, die auf die Feststellung der biologischen Vaterschaft abzielten; hier hat er eine Grenzlinie gezogen. Zwar müsse es möglich sein, die biologische Vaterschaft als Voraussetzung für ein Umgangsrecht festzustellen, wenn dies das Kindeswohl erfordert. ${ }^{72}$ Dies kann aber nicht so weit gehen, dass der biologische Vater den „rechtlichen “ Vater und damit denjenigen, der mit der Mutter zusammenlebt und sich tatsächlich um das Kind kümmert, aus seiner Rolle verdrängt.

Dabei ist anzumerken, dass der Gerichtshof grundsätzlich zurückhaltend ist, bei „nur“ biologischen Vätern, die nie mit ihren Kindern gelebt haben und sie womöglich noch nicht einmal gesehen haben, ein „Recht auf Familie“ anzuerkennen. Originalton Gerichtshof:

„Die biologische Verwandtschaft zwischen dem natürlichen Elternteil und dem Kind, ohne jedes weitere rechtliche oder faktische Element, das die Existenz einer engen persönlichen Beziehung aufzeigen würde, ist unzureichend für einen Schutz nach Art. 8. In der Regel ist das Zusammenleben erforderlich, damit eine Beziehung ein Familienleben darstellt." "73

Zwar sieht der Gerichtshof Ausnahmen dann als möglich an, wenn ein Familienleben beabsichtigt ist, aber ohne Schuld des
Betroffenen nicht verwirklicht werden kann, lässt die Frage bei den biologischen Vätern aber im Ergebnis offen, da zumindest ein Konventionsschutz über das Privatleben erreicht werden kann. ${ }^{74}$

In den Fällen Ahrens und Kautzor hat der Gerichtshof ausgeführt, dass bei Statusfragen, anders als beim Umgangsrecht, ein sehr weiter Ermessensspielraum der einzelnen Mitgliedsstaaten bestehen müsse; hier gebe es auch keinen europäischen Konsens, der eine Orientierung vorgebe. Vor diesem Hintergrund sei eine gesetzliche Regelung, die die Stabilität der rechtlich-sozialen Familie zu schützen bestimmt ist und dem potentiellen Vater kein Recht auf Feststellung der Vaterschaft einräumt, aus der Sicht der Konvention nicht zu beanstanden. ${ }^{75}$

65 Vgl. zur Rechtsprechungsentwicklung und zu den Einflüssen auf die deutsche Gesetzgebung Michael Coester, Sorgerechtliche Impulse aus Straßburg, NJW 2010, S. 482ff; kritisch zur Umsetzung der Straßburger Vorgaben ins deutsche Recht: Lore Maria Peschel-Gutzeit, Der doppelte Vater - Kritische Überlegungen zum Gesetz zur Stärkung der Rechte des leiblichen, nicht rechtlichen Vaters, NJW 2013, 2466 ff; Martin Löhnig, Mareike Preisner, Zur Reichweite des Einflusses der Rechtsprechung des EuGHMR auf das deutsche Kindschaftsrecht, FamRZ 2012, S. 489 ff, Monika Böhm, Dynamische Grundrechtsdogmatik von Ehe und Familie? VdStRL Band 73, 2013, S. 211 ff, S. 245 ff.

66 Ein Beispiel für eine erfolgreiche Beschwerde einer Mutter, die allerdings das Umgangsrecht nach der Scheidung, nicht nach der Trennung in einer nicht-ehelichen Lebensgemeinschaft betrifft, ist etwa der Fall EGMR Urt. v. 28.2.2006, Plasse-Bauer v. Frankreich, Beschwerde-Nr. 21324/02. Die Beschwerde der Mutter ist erfolgreich unter Art. 6 EMRK (nicht unter Art. 8 EMRK), da die Gerichtsentscheidung, die die Umgangsrechte der Mutter nach Übertragung des Sorgerechts auf den Vater festlegt, nicht adäquat durchgesetzt wird; vgl. dazu auch den Kommentar von Pascale Boucaud, Le droit aux contacts familiaux sous le prisme des articles 6 et 8 de la Convention Européenne des droits de l'homme, Revue trimestrielle des droits de l'homme 2007, S. 509-520.

67 EGMR Urt. v. 13.7.2000, Elsholz v. Deutschland, BeschwerdeNr. 25735/94, NJW 2001, 2315-2319; EGMR Urt. v. 8.7.2003, Sommerfeld v. Deutschland, Beschwerde-Nr. 31871/96, EuGRZ 2004, 711.

68 EGMR Urt. v. 3.12.2009, Zaunegger v. Deutschland, BeschwerdeNr. 22028/04, NJW 2010, 501.

69 Vorsicht ist geboten, soweit das „Kindeswohl“ eine von allen Seiten gleichermaßen für ihre Position in Anspruch genommene Größe ist; vgl. dazu die interessante rechtsvergleichende Perspektive bei Admark Moyo, Reconceptualising the ,paramountcy principle': Beyond the individualistic construction of the best interests of the child, African Human Rights Law Journal 2012 (12), S. 142 ff.

70 EGMR Urt. v. 21.12.2010, Anayo v. Deutschland, Beschwerde-Nr. 20578/07, NJW 2011, 3565; EGMR Urt. v. 15.9.2011, Schneider v. Deutschland, Beschwerde-Nr. 17080/07, NJW 2012, 2781; vgl. zustimmend zu dem Urteil im Fall Anayo: Tobias Thienel, Human Rights of Biological Fathers v. Hard and Fast Rules: The Case of Anayo v. Germany, German Yearbook of International Law 53, 2010, S. 963 ff; kritisch zum Fall Schneider mit Blick auf einen überzogenen Individualismus, der gerade nicht im Kindeswohl sei: François Boulanger, Droit de visite et intérêt de l'enfant, Recueil Dalloz 2011, no. 42, 2908.

71 EGMR Urt. v. 3.12.2009, Zaunegger v. Deutschland, BeschwerdeNr. 22028/04, NJW 2010, 501: Verletzung von Art. 8 und 14.

72 EGMR Urt. v. 15.9.2011, Schneider v. Deutschland, BeschwerdeNr. 17080/07, NJW 2012, 2781, Rn. 103: "The question of establishment, in access proceedings, of biological - as opposed to legal - paternity will only arise if, in the special circumstances of the case, contacts between the alleged biological father presuming that he is in fact the child's biological parent - and the child are considered to be in the child's best interest."

73 Anayo v. Deutschland, Beschwerde-Nr. 20578/07, para. 56.

74 EGMR Urt. v. 21.12.2010, Anayo v. Deutschland, BeschwerdeNr. 20578/07, NJW 2011, 3565, Rn. 56 f.

75 EGMR Urt. v. 22.3.2012 Kautzor v. Deutschland, BeschwerdeNr. 23338/09 ; EGMR Urt. v. 22.3.2012 Ahrens v. Deutschland, Beschwerde-Nr. 45071/09. 


\section{4) Internationale Kindsentführungsfälle}

Bei internationalen Kindsentführungsfälle, die den Gerichtshof gleichermaßen häufig beschäftigen, gibt es grundsätzlich zwei Konstellationen: Entweder es klagt der Elternteil, der das Kind entführt hat und aufgrund der Haager Kindsentführungskonvention dazu gezwungen wird, das Kind zurückzubringen, oder aber es klagt der „zurückgebliebene“ Elternteil und macht geltend, die Haager Kindsentführungskonvention werde nicht in adäquater Weise durchgeführt. In beiden Fällen berufen sich die Eltern auf ihr Recht auf Familienleben. Zu Verwerfungen hat die Rechtsprechung des Gerichtshofs in der ersten Fallgruppe mit dem Urteil Neulinger-Shuruk v. Schweiz ${ }^{76}$ geführt, da darin erstmals in der Rückführung, würde sie denn stattfinden, eine Konventionsverletzung gesehen und damit zugunsten der Mutter, die das Kind entführt hatte, entschieden worden war. Problematisch war dies insbesondere im Hinblick darauf, dass hohe Anforderungen an die Überprüfung des Falles durch das für die Durchführung der Haager Konvention zuständige Gericht gestellt worden waren, die, so die Kritik, mit der beschleunigten Behandlung derartiger Fälle nur schwer vereinbar wären. In der Entscheidung X. v. Lettland ${ }^{77}$, die ein entsprechendes Szenario betraf, wurde versucht, die hier aufgrund unterschiedlicher Interpretationen bestehenden Probleme zu klären. Wichtig ist in dieser Rechtsprechungslinie auch die Entscheidung Povse v. Österreich, ${ }^{78}$ in der von den erstinstanzlichen österreichischen Gerichten bis zum EuGH in Luxemburg alle denkbaren Instanzen angerufen worden waren, bevor der Fall in Straßburg landete. Dort wurde der Fall für unzulässig erklärt und insoweit ein Gleichlauf der Entscheidungen der beiden obersten europäischen Gerichte in Luxemburg und Straßburg hergestellt. Leider ist „mehr“ bei diesen Fällen nicht „besser“; umso mehr um das Kindeswohl gestritten wird, umso mehr sind die Kinder Verlierer.

Kindsentführungsfälle betreffen die Rechte von Müttern ebenso wie von Vätern, deren Rolle als Erzieher insoweit grundsätzlich als gleichberechtigt gesehen wird. Es mag einer soziologischen Studie vorbehalten bleiben herauszuarbeiten, inwieweit die Entscheidungen dennoch von Vorurteilen oder zumindest vorschnellen Urteilen beeinflusst sind. Nach Straßburg sind als Beschwerdeführer sowohl Männer als auch Frauen gekommen. Und sowohl Männer als auch Frauen haben am EGMR obsiegt.

\section{IV) Mehrwert internationaler Streitentscheidung?}

Die Bedeutung der Rechtsprechung zu den Defiziten staatlicher Schutzgesetzgebung gegen Zwangsarbeit, sexuellen Missbrauch und häusliche Gewalt sowie zum besonderen Schutz von Frauen in Ausweisungsfällen dürfte außer Frage stehen. Allerdings ist es legitim, bei Drittwirkungsfällen wie den allgemeinen familienrechtlichen Fällen zu fragen, welchen Mehrwert hier ein derartiger internationaler Streitbeilegungsmechanismus haben kann. ${ }^{79}$

Im Gegensatz zu nationalen Gerichten hätten internationale Gerichte, wie etwa der EGMR, die Möglichkeit, stereotype Annahmen beiseite zu schieben, so behauptet Oliver Gerstenberg in I-Con im Jahre 2010 ${ }^{80}$ Er bezieht sich dabei auf den Fall Anayo, den er als ,groundbreaking “ mit Blick auf das Verständnis von sozialer und biologischer Elternschaft ansieht.
"Courts can help provide, in situations of deep and socially divisive conflict, an argumentative setting where all participants appear, procedurally, as equals and are required to defend their interpretations of law with reference to reasons that are capable of being acknowledged by all others involved." ${ }^{81}$ Dass gerade im Familienrecht Konflikte, die besonders tiefgehend, ja existentiell sind, bestehen, ist sicherlich richtig. Bietet der EGMR zur Lösung jener Konflikte einen adäquaten prozeduralen Rahmen? Werden die Auseinandersetzungen auf einer „rationaleren“ - sofern es einen derartigen Komparativ geben mag - Ebene geführt, stoßen die Entscheidungen des EGMR im Familienrecht auf Akzeptanz, auf mehr Akzeptanz als die Entscheidungen anderer Gerichte?

Ein erster bemerkenswerter Aspekt ist, dass die Konflikte „gekippt“ und von der horizontalen Ebene auf die vertikale Ebene verlagert werden. Vor den nationalen Gerichten streiten die Väter gegen die Mütter oder die Mütter gegen die Väter. In Straßburg klagt der Einzelne gegen den Staat, da dieser in die jeweiligen Rechte zu Unrecht eingegriffen habe oder seinen positiven Verpflichtungen nicht nachgekommen sei. ${ }^{82}$ Schon psychologisch ist es etwas anderes, ob ein Rechtsstreit von „Müller gegen Müller“ oder von „Müller gegen Deutschland“ geführt wird; das persönliche Problem bekommt auf diese Weise plötzlich eine grundsätzliche, weit über den Einzelfall hinausragende allgemeingültige Dimension. Damit werden Beschwerdeführer von Einzelkämpfern zu Vorkämpfern für die Rechte der „Erniedrigten und Beleidigten“ im Sinne Dostojewskijs.

Nun ist der Befund aus der Konventionsrechtsprechung, geht es um die Rechte der Frauen, aber ein erstaunlicher: Zwar waren es Frauen, die mit ihren Beschwerden auf ihre besondere Verletzbarkeit als Opfer von Zwangsarbeit, sexuellem Missbrauch und unmenschlicher Behandlung aufmerksam gemacht haben. Soweit es aber um den Bereich Familienrecht im weiteren Sinn einschließlich sozialrechtlicher Absicherungen ging, waren es in aller Regel die Männer, die gegen den Frauen vorbehaltene Privilegien gekämpft und damit erreicht haben, dass bestimmte gesellschaftliche Vorurteile gerichtlich - und damit gewissermaßen mit Tonverstärker - für unhaltbar erklärt wurden. Manchmal hat dies Frauen genützt, manchmal, wie etwa bei der Rechtsprechung zu den biologischen Vätern, im Einzelfall aber auch zu Einschränkungen, vielleicht sogar zu Härten geführt.

76 EGMR Urt. v. 6.7.2010, Neulinger-Shuruk v. Schweiz, BeschwerdeNr. 41615/07.

77 EGMR Urt. v. 26.11.2013, X. v. Lettland, Beschwerde-Nr. 27853/09.

78 EGMR Urt. v. 18.6.2013, Povse v. Österreich, Beschwerde-Nr. 3890/11, FamRZ 2013, 1793

79 Vgl. dazu allgemein Andreas von Staden, The democratic legitimacy of judicial review beyond the state: Normative subsidiarity and judicial standards of review, I-CON 2012, S. 1023-1049.

80 Oliver Gerstenberg, Negative/positive constitutionalism, „fair balance", and the problem of justiciability, I-Con 2012, S. 904 ff.

81 Gerstenberg (Fn. 80), S. 925.

82 Zu den positiven Verpflichtungen des Staates bei familienrechtlichen Auseinandersetzungen vgl. Nathalie Mathieu, Séparation des parents et garde d'enfant - Le point sur la jurisprudence de la Cour européenne des droits de l'homme, Rev. Trim. Dr. H. 93/2013, S. 39 ff. 
Ein zweiter besonderer Aspekt lässt sich beobachten, der ebenfalls mit der Verlagerung der Auseinandersetzung von der horizontalen auf die vertikale Ebene zusammenhängt: der eigentliche Gegner bzw. die eigentliche Gegnerin wird unsichtbar. Macht ein Vater geltend, der Staat habe sein Recht auf Familienleben verletzt, weil ihm die nationalen Gerichte in konventionswidriger Weise kein Umgangsrecht mit seinem Kind eingeräumt hätten, so ist die Mutter im Verfahren nicht mehr sichtbar. Ihre Argumente, ebenso wie die Argumente des Kindes, muss der Staat übernehmen. Daraus resultieren Verwerfungen. So hat im Fall Anayo die Mutter der Zwillinge ihren Vortrag, die Stabilität ihrer Familie sei gefährdet, wenn sie dem biologischen Vater ihrer Kinder ein Umgangsrecht einräumen müsse, als „third party intervener“, als Drittintervenientin, vortragen müssen. ${ }^{83}$

Und ein dritter Gesichtspunkt in diesem Zusammenhang ist schließlich noch wichtig. Der Streit geht nicht mehr darum, wer im Recht und wer im Unrecht ist. Vielmehr steht eine Menschenrechtsverletzung im Raum und damit etwas, was per se verwerflich ist. Der Vorwurf ist gegen den Staat gerichtet, der sich verteidigt, um mit einer menschenrechtlich reinen Weste dazustehen. Wer seinen Prozess in Straßburg gewinnt, ist - mit Blick auf das auf nationaler Ebene geführte Verfahren - nicht mehr nur „Verlierer“, sondern „Opfer“. Es ist eine unverkennbar andere Dramaturgie, mit der auf diese Weise um familienrechtliche Positionen gestritten wird.

In diesem geänderten Rahmen ist der Mehrwert der Urteile des EGMR auszumachen.

Ein Mehrwert liegt sicherlich darin, dass das jeweilige Problem von außen betrachtet wird. Sechs von sieben Richtern in der Kammer, 16 von 17 in der Großen Kammer kommen nicht aus dem Land, in dem der Konflikt sich abgespielt hat. Der Perspektivwechsel ist zweifellos hilfreich und vermag Schwachstellen gerade dann aufzudecken, wenn man aus der Innenansicht betriebsblind ist.

Zum anderen wird die entsprechende Fragestellung in den europäischen Rahmen gestellt. Insbesondere bei innovativen Fragestellungen fragt der Gerichtshof nach einem „europäischen Konsens“, um auszuloten, inwieweit ein Spielraum für unterschiedliche Lösungen besteht. Es ist vielfach kritisiert worden, dass dieser auf Rechtsvergleichung basierende Schluss von einem Sein zu einem Sollen problematisch ist, auch wenn der Gerichtshof hier inzwischen eine differenzierte Methodik entwickelt hat und versucht, auch abweichenden Ansätzen kulturspezifisch gerecht zu werden. ${ }^{84}$ Aber für die Betroffenen bedeutet es doch, dass sie mit ihren Fragen und Problemen nicht alleine sind und bei den Lösungen der Konfliktfälle ein europäischer Gleichlauf zu erkennen ist.

Und schließlich, so komplex einzelne Argumentationsmuster und Herleitungen aus dem case law sein mögen, die Elle, mit der der Gerichtshof misst, ist, anders als nationales Gesetzesrecht, mit letztlich für das Familienrecht nur zwei wirklich relevanten Artikeln, Art. 8 und Art. 14 EMRK, für Nicht-Juristen gut zugänglich. Auch die Grundfragen, auf die der Gerichtshof die Sachverhalte zuspitzt, sind leicht vermittelbar. Denn im Grunde wird bei jedem Eingriff in ein Recht gefragt, ob er „notwendig in einer demokratischen Gesellschaft" ist. Und darauf gibt der Gerichtshof in jedem Einzelfall eine klare und endgültige
Antwort: Ja oder Nein, das Nein in aller Regel mit einer Schadensersatzzahlung verbunden.

Dass dennoch nicht immer der lange Weg nach Straßburg den wirklichen Abschluss eines Konflikts bedeutet, hat die Leidensgeschichte des Herrn Görgülü veranschaulicht, der vom EGMR zum Amtsgericht und von dort wieder ans Bundesverfassungsgericht laufen musste, um sein von der Mutter des Kindes ohne sein Wissen zur Adoption freigegebenes Kind sehen zu können. ${ }^{85}$ Der Name Görgülü steht inzwischen als juristisches Kürzel für die Auseinandersetzung um die Implementierung der Entscheidungen des EGMR ins deutsche Recht, ähnlich wie der Name Marckx mit der Idee einer Neuausrichtung der Rechtsprechung des Gerichtshofs in den 1970er Jahren verbunden ist. Hinter all diesen Namen aber stehen Geschichten, mehr noch, Schicksale.

Und so sei zum Schluss noch einmal der Name „Marckx“ herausgegriffen. Paula Marckx war 47 Jahre alt, als sie im Jahr 1973 feststellte, dass sie schwanger war ohne zu wissen, von wem. Es war vielleicht ihrer journalistisch spitzen Feder zu verdanken, dass ihr Fall als erster familienrechtlicher Diskriminierungsfall am Straßburger Gerichtshof verhandelt wurde. Nachdem ihr auf ihre erste Anfrage hin erklärt worden war, sie als Mutter sei nicht klageberechtigt, schrieb sie einen Brief, der mit folgenden Worten begann: „Meine Herren, ich bin ein zehn Monate altes Baby. Ich hoffe von ganzem Herzen [...]“ - und der Brief endete - „[...], dass ein Baby in meinem Alter auf eine Institution wie die Ihre vertrauen kann, um seine Rechte zu schützen. “86

Der Gerichtshof hat die Rechte von Paula und Alexandra Marckx geschützt, sie haben ihren Prozess in Straßburg gewonnen. Hätte sich damals die abweichende, mit Vehemenz vorgetragene Meinung des britischen Richters Fitzgerald durchgesetzt, der das Familienrecht, und mag es auch diskriminierend sein, als nicht von der Konvention umfasst ansah, ${ }^{87}$ so hätten bei den Auseinandersetzungen um Rollen und Rechte von Frauen in den europäischen Staaten, insbesondere auch in Deutschland, entscheidende Reformanstöße von außen gefehlt.

83 EGMR Urt. v. 21.12.2010, Anayo v. Deutschland, BeschwerdeNr. 20578/07, para. 54; NJW 2011, 3565; vgl. dazu Almut WittlingVogel, Die Beteiligung Dritter in Verfahren vor dem Europäischen Gerichtshof für Menschenrechte - ein Plädoyer für die Besserstellung der in eigenen Rechten Betroffenen, in: S. LeutheusserSchnarrenberger, V. Recht auf Menschenwürde. 60 Jahre Europäische Menschenrechtskonvention, Tübingen 2013, S. 241-256.

84 Luzius Wildhaber, Arnaldur Hjartarson, No Consensus on Consensus? The Practice of the European Court of Human Rights, HRL 2013 , S. 248-263, Antje von Ungern-Sternberg, Die Konsensmethode des EGMR. Eine kritische Bewertung mit Blick auf das völkerrechtliche Konsens- und das innerstaatliche Demokratieprinzip, Archiv des Völkerrechts 2013, S. 312-336, Angelika Nußberger, Europäischer Konsens als rechtliche Fiktion, in: Beckmann, Mansel, MatuscheBeckmann (Hg.), Weitsicht in Versicherung und Wirtschaft. Festschrift für Ulrich Hübner, Heidelberg 2012, S. 717-731.

85 EGMR Urt. v. 26.2.2004, Görgülü v. Deutschland, BeschwerdeNr. 74969/01, NJW 2004, 3397-3401; BVerfG E 111, 307-322, BVerfG, 1 BvR 1664/04 v. 5.4.2005; BVerfG, 1 BvR 2790/04 v. 10.6.2005 NJW 2008, S. 223-227; Vgl. zu dem Fall Gertrude Lübbe-Wolff, Humboldt Forum Recht 2006, S. 138 ff.

86 Zitiert nach Michael D. Goldhaber, A People's History of the European Court of Human Rights, 2007, S. 17.

87 EGMR Marckx v. Belgien, Beschwerde-Nr. 6833/74, NJW 1979, 2449; abweichende Meinung des Richters Fitzgerald. 